\title{
Universiteit
}

Leiden

The Netherlands

\section{Graphical analysis and experimental evaluation of Saccharomyces cerevisiae P_TRK1|2 and P_BMH1|2 promoter region}

Gerber, S.; Hasenbrink, G.P.H.; Hendriksen, W.; Heusden, G.P.H. van; Ludwig, J.; Klipp, E.; ... ; Wallach, I.

\section{Citation}

Gerber, S., Hasenbrink, G. P. H., Hendriksen, W., Heusden, G. P. H. van, Ludwig, J., Klipp, E., \& Lichtenberg-Fraté, H. (2010). Graphical analysis and experimental evaluation of Saccharomyces cerevisiae P TRK1|2 and P BMH1|2 promoter region. Genome Informatics 2009, $2 \overline{2}, 11-20$. doi:10.1142/9781848165786_0002

Version: $\quad$ Not Applicable (or Unknown)

License: $\quad$ Leiden University Non-exclusive license

Downloaded from: $\quad$ https://hdl.handle.net/1887/61222

Note: To cite this publication please use the final published version (if applicable). 


\title{
GRAPHICAL ANALYSIS AND EXPERIMENTAL EVALUATION OF SACCHAROMYCES CEREVISIAE $P_{T R K_{1 \mid 2}}$ AND $P_{B M H_{1 \mid 2}}$ PROMOTER REGION
}

\author{
SUSANNE GERBER ${ }^{1}$ \\ gerber@molgen.mpg.de
}

\author{
GUIDO HASENBRINK ${ }^{2}$ \\ uzs6br@uni-bonn.de
}

PAUL VAN HEUSDEN ${ }^{3}$

G.P.H.van.Heusden@biology.leidenuniv.n1

\author{
WOUTER HENDRIKSEN ${ }^{3}$ \\ w. t. hendriksen@biology.leidenuniv.nl \\ JOST LUDWIG ${ }^{2}$ \\ jost.ludwig@uni-bonn.de
}

EDDA KLIPP 1

edda.klipp@rz.hu-berlin.de
HELLA LICHTENBERG-FRATÉ ${ }^{2}$

h.lichtenberg@uni-bonn.de

\author{
${ }^{1}$ Group of Theoretical Biophysics, MNF I,Humboldt University of Berlin, \\ Invalidenstrasse 42, 10115 Berlin, Germany \\ ${ }^{2}$ University of Bonn, IZMB, Kirschallee 1 - D 53115 Bonn , Germany \\ ${ }^{3}$ Section Molecular and Developmental Genetics, Institute of Biology, Leiden \\ University,Sylviusweg 72, 2333 BE Leiden, The Netherlands
}

\begin{abstract}
We designed a simple graphical presentation for the results of a transcription factor (TF) pattern matching analysis. The TF analysis algorithm utilized known sequence signature motifs from several databases. The graphical presentation enabled a quick overview of potential TF binding sites, their frequency and spacing on both DNA strands and thus straight forward identification of promising candidates for further experimental investigations. The developed tool was applied on in total four Saccharomyces cerevisiae gene promoter regions. The selected differentially expressed genes belong to functionally different families and encode duplicate functions, TRK1 and TRK2 as ion transporters and $\mathrm{BMH} 1$ and $\mathrm{BMH} 2$ as multiple regulators. Output evaluation revealed a number of TFs with promising differences in the promoter regions of each gene pair. Experimental investigations were performed by using corresponding TF yeast mutants for either phenotypic analysis of ion transport mediated growth or expression analysis of BMH1,2 genes. Upon phenotypic testing one TF mutant exhibited severely impaired growth under non-permissive conditions. This TF, Mot3p was identified as of most abundant potential binding sites and distinctive patterns among the TRK promoter regions.
\end{abstract}

Keywords: Saccharomyces cerevisiae; promoter region; graphical analysis tool.

\section{Introduction}

In the post-genome era the task of regulatory elements prediction and in particular promotor region analysis has received increasing interest and a considerable number of algorithms, tools and analysing strategies was developed. However, despite many useful approaches signal finding such as pattern discovery in DNA sequences is still a fundamental problem in molecular biology with such important applications as locating regulatory sites and drug target identification. Most signals in DNA sequences are indeed complex or not yet discovered, such that there is a lack of 
good models or reliable algorithms for their authentic recognition. For instance, some transcription factor binding sequences are injective but the majority show a rather high degree of wobbling which means in mathematical terms they show a bijective behaviour. Concerning the model organism $S$. cerevisiae the currently available tools like AlignACE [10], Consensus [6], TESS [16, 27], TRANSFAC [23] or Genomatix [25] differ from each other basically in their definitions of what constitutes a motif, what constitutes statistical overrepresentation of a motif and the methods used to identify statistically overrepresented motifs (for a review see [18]). All these tools differ enormous in their binding site predictions or recognize motifs that are not even specific for the queried organism, e.g. mammalian TF motifs in the promoter regions of S.cerevisiae. In all cases the graphical presentation of positive hits on both strands is a highly condensed and information-wise insufficient image. An additional obstacle for wet lab scientists might be the fact that they are offered little guidance in the choice among these tools. Since there have been published only a few assessments or reviews of some of these motif discovery tools ( [15] and [18]) and it is virtually impossible to decide on objective grounds of whether the results of the tool (A) are better or more plausible than those of tool (B). In conjunction with a study focusing on cation homeostasis, ion transporters and their potential regulators in yeast we collated a dataset of TFs (sequence signature motifs) known as functional in $S$. cerevisiae. The challenge was to provide on the basis of this comprehensive dataset a bioinformatical analysis that should reveal first indications of potential TFs for specific genes, or their upstream regulating sequences respectively, involved in cation transport or their possible regulators. The analysis emphasized on frequency, spacing and accuracy of match and presented the results in a graphical format enabling straight forward visual inspection and decisions on potentially promising candidates for further experimental investigation.

As examples for an explorative approach the promoter regions of the TRK1 and TRK2 and BMH1 and BMH2 were selected. These genes encode duplicated functions in yeast, are differentially expressed and highly regulated and appeared thus as reasonable candidates for a comparative promoter region analysis. The first ones are components of the potassium transport system $[3,11,12,24]$, and latter, belonging to the group of 14-3-3 proteins, act as multiple regulators. 14-3-3 proteins are a family of highly conserved proteins and have been identified in all eukaryotic species investigated. S. cerevisiae has two genes encoding 14-3-3 proteins, BMH1 (major isoform) and BMH2, and these proteins are involved in various cellular processes [21]. Numerous studies revealed that expression of BMH1 and BMH2 is affected by different circumstances (for a review see [22]). Expression of BMH1 and $\mathrm{BMH} 2$ might therefore be tightly regulated by transcription factors. 


\section{Material and Methods}

\subsection{Bioinformatical Analysis}

We collected a data set of all available TFs known to be functional in yeast within the literature documented binding sites. For collection, we parsed the YEASTRACT database $[17,28]$ (Yeast Search for Transcriptional Regulators And Consensus Tracking) that presently contains 30990 regulatory associations between the yeast genes based on more than 1000 bibliographic references [17]. Each regulation has been manually annotated after examination of the relevant references. Further information about each yeast gene was obtained from the Saccharomyces Genome Database (SGD) [9], Regulatory Sequence Analysis Tools (RSAT) [20] and Gene Ontology (GO) Consortium [19]. The assembled dataset for this study contained the description of 288 specific and in the literature documented DNA binding sites for a group of 108 transcription factors.

The analysis was performed by implementing a Matlab [26] program that imports a promotor sequence in fasta-format as well as the data set of TFs with their dedicated potential binding sequences. If the TF binding sequence contains IUPAC codes other than one of the four nucleotide the program will identify all possible matches. For example, the symbol ' $R$ ' in a sequence represents a purine at this position (either $\mathrm{G}$ or $\mathrm{A}$ ) and the algorithm would accomplish a pattern search for each sequence on the promotor. For each match the start and stop position on the query promotor promoter sequence is stored, as well as the probability of a match of this sequence by just chance. If more than one match of the investigated sequence is identified, the distances between all possible binding sites of this TF are calculated. At the end of the analysis the user will receive a table with the information of every potential binding sequence combined to the following documentation: the names of the tested TFs and the related sequences; the average likelihood for every sequence to bind on this promoter just by chance; for succesfull matches the start and stop positions on both strands and, in case that more than one succesful match was found, the distances between all these potential binding sites. Furthermore - as a highlight - a graphical presentation of all potential binding sites on the forward and backward strands for every promotor region is a key add on.

\subsection{Yeast Strains, Media and Growth Tests}

\section{Experiment 1}

Haploid $S$. cerevisiae wild type, potassium transporter and TF mutant strains (BY4741; [2]; Saccharomyces Genome Deletion Project) were used throughout this study as listed in Table 1 . Strains were grown aerobically at $28^{\circ} \mathrm{C}$ with rotational shaking at $250 \mathrm{rpm}$ in liquid amino acid, ammonium sulphate and potassium free YNB-F media (CYN7505, Formedium). The medium was supplemented with a complete supplement mixture (DBSM225, Formedium), ammonium sulphate, 2\% D-glucose and, for precultures $50 \mathrm{mM} \mathrm{KCl}$. For test cultures $\mathrm{KCl}$ was added to 
concentrations as indicated. All medium components were dissolved in ultrapure MilliQ water and $\mathrm{pH}$ was adjusted to 5.8 with ammonium hydroxide. Stationary phase cells were washed three times in MilliQ water and diluted in fresh YNB-F medium to a start optical density OD600 of 0.4 (pathlength $1 \mathrm{~cm}$, Pharmacia Ultrospec 2000 Spectrophotometer, corresponding to $6 \times 106$ cells per mL) with respective $\mathrm{KCl}$ concentration. Growth assessment was obtained by photometric determination of the turbidity at $600 \mathrm{~nm}$ in 15-min intervals in transparent 96-well microtiter plates using a microplate reader (Tecan, Infinite $\mathrm{F} 200$ ) with $28^{\circ} \mathrm{C}$ incubation and constant agitation for $17 \mathrm{~h}$. Growth calculation and statistical analysis was performed by integral determination (approximation of the area under the growth curves) obtained within $17 \mathrm{~h}$ incubation according to [5]. Subsequently values for growth were normalized to those of wild type strain values at $50 \mathrm{mM}$ $\mathrm{KCl}$.

Table 1: Selected transcription factors for experimental analysis

\begin{tabular}{lrrr|rrr}
\hline $\begin{array}{l}\text { Transcription Factor } \\
\text { matches }\end{array}$ & foreward & $\begin{array}{r}\text { TRK1 } \\
\text { reverse }\end{array}$ & total & foreward & $\begin{array}{r}\text { TRK2 } \\
\text { reverse }\end{array}$ & total \\
\hline Fkh1p & $4 *$ & $5 *$ & 9 & $3 *$ & 1 & 4 \\
Fkh2p & $4 *$ & $5 *$ & 9 & $3 *$ & 1 & 4 \\
Mot3p & $2 *$ & $11 *$ & 13 & 6 & 1 & 7 \\
Msn2p & & 1 & 1 & 3 & & 3 \\
Msn4p & 3 & 1 & 1 & 3 & 6 & 3 \\
Stb5p & 3 & 4 & 4 & 6 & 10 \\
\hline
\end{tabular}

Note: * Total number of hits with more than one possible binding sequence

\section{Experiment 2}

In a second experimental scenario the $S$. cerevisiae BY4743 wild type $M A T a / \alpha \quad$ his $3 \Delta 1 /$ his $3 \Delta 1$ leu $2 \Delta 0 /$ leu $2 \Delta 0 \quad$ LYS $2 /$ lys $2 \Delta 0 \quad$ met $15 \Delta 0 / M E T 15$ ura $3 \Delta 0 /$ ura $3 \Delta 0$ ) and transcription factor mutant (Saccharomyces Genome Deletion Project [4]) cells were grown exponentially in YPD broth to an $O D_{620}$ of 0.6-0.8. The cells were harvested by immediately freezing with liquid nitrogen. Total RNA was isolated using the RiboPure ${ }^{T M}$-Yeast Ambion RNA isolation kit (Ambion) according to the manual. This procedure included a DNase treatment to breakdown all chromosomal DNA. Subsequently, cDNA was produced using the Superscript III Reverse Transcriptase (Invitrogen), according to the manual. cDNA was diluted two times and $1 \mu \mathrm{l}$ was used as a template for qPCR using the DyNAmo ${ }^{T M}$ Probe qPCR Kit (Finnzymes, Bioke). ACT1, BMH1, and BMH2 relative gene expression was measured on the Chromo4 ${ }^{T M}$ Real-Time Detector (Biorad) using Opticon3 motion software (Biorad). The oligonucleotide primers used in this study are listed in Table 2. BMH1 and $\mathrm{BMH} 2$ gene expression ratios were 
calculated using the the $\Delta \Delta \mathrm{C}(\mathrm{t})$ method [13], and these ratios were normalized to ACT1 expression and normalized to wild type BMH1 and BMH2 expression. This procedure was performed for two biological duplicates and expression was measured in triplicate and in duplicate for every sample.

Table 2: Oligonucleotide primers

\begin{tabular}{lll}
\hline Primer name & Sequence 5'-3' & Target \\
\hline WH11 & AGAGTTGCCCCAGAAGAACA & ACT1 \\
WH12 & GGCTTGGATGGAAACGTAGA & ACT1 \\
WH29 & CGTGCTAGACTCCCACTTAATT & BMH1 \\
WH30 & GGCCTTTTCTCTAGCATCG & BMH1 \\
WH15 & GTTAGATTCTCATTTAATCCCTTCT & BMH2 \\
WH16 & ATAAGCCTCCAAAGAGGAGTTG & BMH2 \\
\hline
\end{tabular}

\section{Results}

The complete resulting output table for all 288 tested TF sequence motifs for genes TRK1,2 and for BMH1,2 are accessible in the supplementary material section, as well as the created graphics. As an example the graphical output of the TRK1 promotor analysis is given in Fig. 1. Out of the entire output list promising candidates for experimental investigations were selected along the following criteria: Sequence unambiguousness in the pattern matching of TF sequence signature to the DNA; the frequency of hits and significant differences in the frequency of hits between the analysed promoter regions of the gene pairs; a rational spacing between the potential TF binding sites (predicted potential binding sites that were at close proximity would constrain each other and were thus devaluated).

Consequently for the TRK1,2 genes 10 potential TFs were selected for further experimental analysis and for the Bmh1,2 genes 7 TFs. Since in the cation homeostasis project we are also interested in other cation transport systems of S. cerevisiae the promoter regions of the ENA1, TOK1, PHO89, NHA1 and PMA1 genes were subjected to computational $\mathrm{TF}$ sequence signature analysis including graphical presentation. However, these potentially promising transcription factor candidates have not yet been experimentally investigated. Calculations and graphics are provided in in supplementary material.

\section{Experiment 1}

In $S$. cerevisiae tight control and modulation of the intracellular $\mathrm{K}^{+}$concentration (cellular $K^{+}$homeostasis) and corresponding ion fluxes is crucial for cell metabolism, surveillance and growth. Two closely related plasma membrane localised $K^{+}$translocation systems, TRK1p and TRK2p mediate $K^{+}$uptake to 


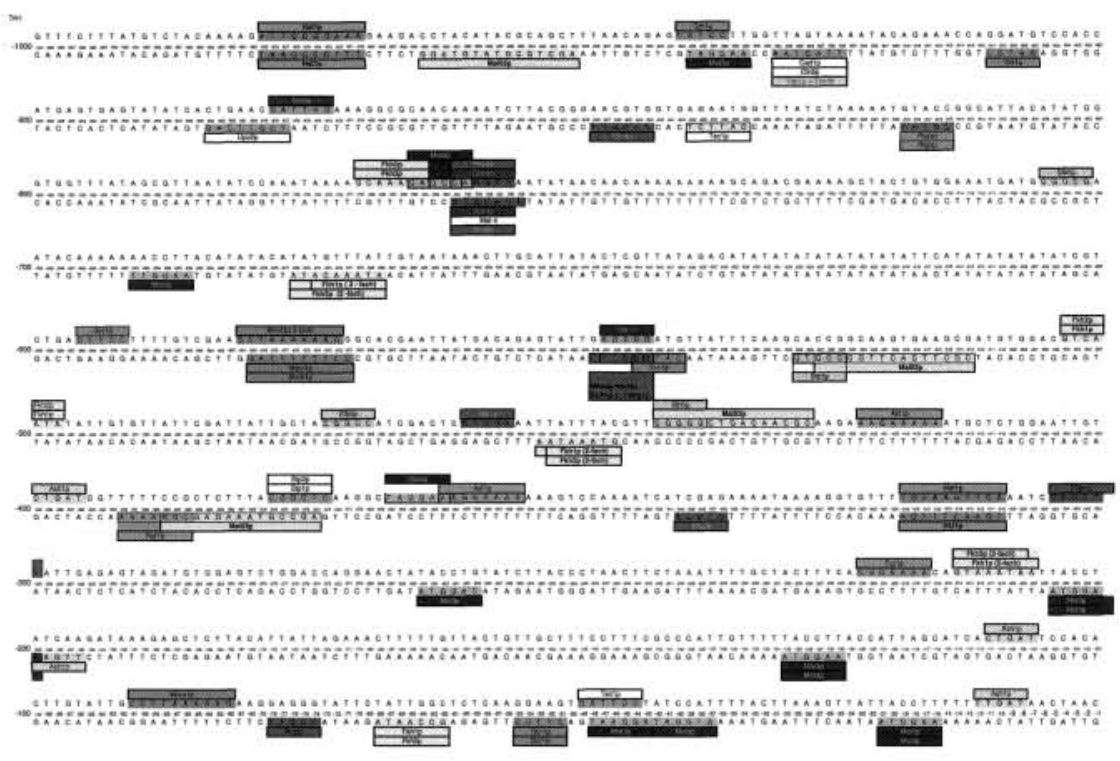

Fig. 1: Graphical analysis of TRK1 promotor regions.

maintain a high intracellular potassium concentration. S. cerevisiae strains devoid of TRK1 $(\Delta \mathrm{TRK} 1)$ or TRK1 and TRK2 $(\Delta \mathrm{TRK} 1,2)$ are defective in potassium uptake and exhibit impaired growth on limited external potassium con-centrations. From the potassium transporters TRK1 and TRK2 promoter region binding site analysis in total 10 potential TFs were obtained. Selection of these TFs or respective mutants as potentially important for further experimental analysis followed the simple rationale of clear frequency and pattern difference within the two promoter regions. Of these, four TF mutant strains are reported as not viable (Saccharomyces Genome Database, SGD). The methodological approach for the remaining six TF mutants was set accordingly: Will the lack of these TFs influence the TRK-system mediated potassium uptake detectable as growth differences under limited potassium conditions? The mutant yeast strains $\Delta \operatorname{mot} 3, \Delta \operatorname{msn} 2, \Delta \operatorname{msn} 4$, $\Delta$ stb5, $\Delta$ fkh1, $\Delta$ fkh2 carry disruptions in the respective TF genes. The growth of these strains was compared to that of $S$. cerevisiae wild type, $\triangle T R K 1$ und $\Delta T R K 1,2$ under potassium limited and permissive conditions (Fig. 2). At $10 \mu \mathrm{M} \mathrm{KCl}_{\text {ext }}$ none of the strains did grow. At $1 \mathrm{mM} \mathrm{KCl}_{\text {ext }}$ considerable differences between the strains were observed. The $\Delta$ TRK1 strain, expressing only the TRK2 transporter grew only 


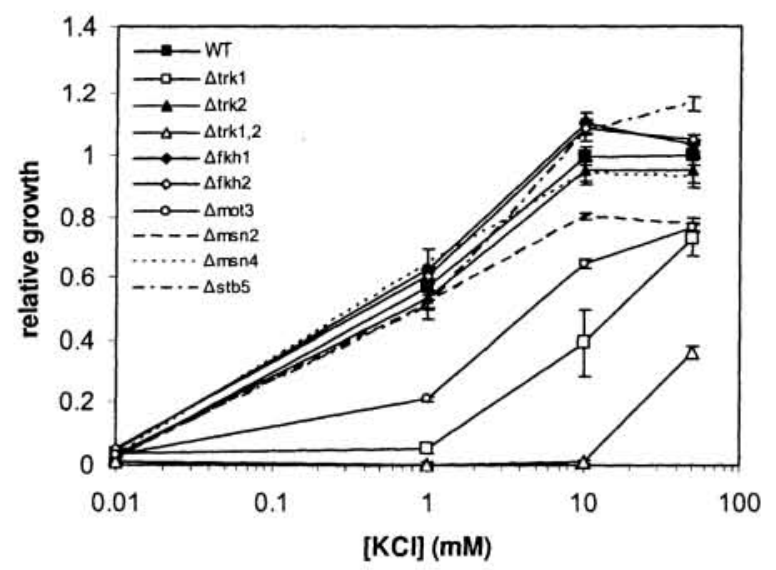

Fig. 2: Analysis of the survey data from experiment 1.

marginally above inoculum. In contrast, the $\Delta$ TRK2 strain, expressing solely TRK1 grew comparable to the wild type. The double $\Delta$ TRK1,2 mutant showed no growth at all. These results were consistent with previously published data $[1,5]$. The strains with disruptions in the transcriptions factors FKH1/2, MSN2/4 und STB5 exhibited growth comparable to the wild type. However, growth of the TF mutant $\Delta$ mot3 strain was considerably reduced compared to the wild type and the $\Delta T R K 2$ strain $(\sim 60 \%)$. At $10 \mathrm{mM} \mathrm{KCl}$ ext the potassium transporter mutant $\triangle T R K 1$, $\Delta \mathrm{TRK} 1,2$ and the $\Delta \operatorname{mot} 3$ strains showed with $51-64 \%$ and $20 \%$ respectively, reduced growth compared to the wild type. Mutant strains $\Delta \mathrm{fkh} 1 / 2$ and $\Delta$ stb5 exhibited slightly enhanced growth ( 10\%). At $50 \mathrm{mM} \mathrm{KCl}_{\text {ext }}, \Delta \mathrm{TRK1}, 2, \Delta \mathrm{TRK} 1, \Delta \operatorname{mot} 3$, $\Delta \mathrm{msn} 2$ showed impaired growth of $64 \%, 27 \%, 24 \%$ and $22 \%$ compared to the wild type. Strains $\Delta \mathrm{fk} 1 / 2$ and $\Delta$ stb5 showed similar growth as the wild type.

\section{Experiment 2}

Using mutants for TFs predicted to bind to one or both of the upstream regions of BMH1 and BMH2 (see suplementary material of the bioinformatical output), we tested the effect on expression during exponential growth in YPD medium. The used strains were null mutants of MSN2, RTG1, WAR1, RLM1, ASH1, FKH1, or GSM1. Grown in YPD medium, we did not observe any differences in growth of these strains. Compared to wild-type BY4743, BMH1 expression did not significantly change in the tested mutants and, hence, is probably not be regulated under the tested condition (Fig. 3A). The same was observed for BMH2 expression (Fig. 3B). We used the arbitrary twofold up- or downregulation as cut-off value for differential expression. However, subtle regulation by these transcription factors (i.e., fold change not higher than 2 and not lower than 0.5) cannot be excluded. In addition, other TFs may take over the regulatory role of the ones tested in this study. For 
instance, partial redundancy has been described for several TFs, e.g., MSN2/MSN4 and FKH1/FKH2 $[8,14]$. In conclusion, we were not able to show an effect on regulation of $\mathrm{BMH} 1$ and $\mathrm{BMH} 2$ in the TFs mutants tested in this study.
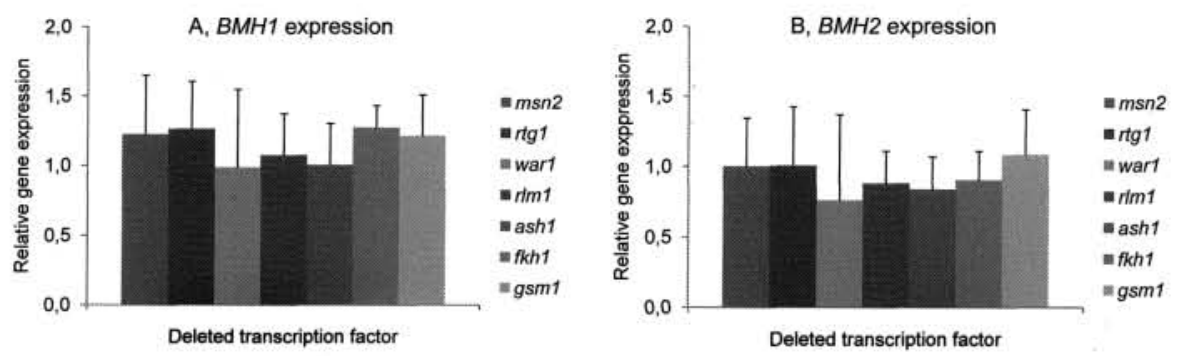

Fig. 3: Relative gene expression of BMH1 (A) and BMH2 (B) in BY4743 $\Delta / \Delta$ msn2, $\Delta / \Delta \operatorname{rtg} 1, \Delta / \Delta$ war1, $\Delta / \Delta \operatorname{rlm} 1, \Delta / \Delta \operatorname{ash} 1, \Delta / \Delta$ fkh1, $\Delta / \Delta$ gsm1 measured by real-time PCR. Gene expression was normalized to ACT1 expression and expression of BMH1 and BMH2 in BY4743 wild-type.

\section{Discussion}

\section{Experiment 1}

Six potential transcription factors with differential frequency and binding site patterns in the potassium transporters TRK1 and TRK2 promoter regions were subjected to growth tests of respective yeast mutant strains for potassium dependent growth. Disruption of the Fkh1/2 TFs ( 9 potential binding sites in TRK1 and 4 in TRK2 promoter region) caused at limiting potassium conditions no reduction in mitotic growth. Both TFs are important in the cell cycle, FKH1 as negative regulator and Fkh2 as positive regulator within the elongation process during transcription. Deletion of the negative regulator caused slight enhancement of growth under permissive conditions. For the Msn2/4p stress transcription factors (ratio TRK1:TRK2 of 1: 3) on TRK1 region only one potential site on the reverse strand was found whereas on the TRK2 region three potential sites were found on the forward strand. Deletion of these stress TFs had minor consequences on the $\mathrm{K}^{+}$ growth phenotype. We conclude that Msn2/4 TFs are inferior for the upregulation of TKR transporters under $K^{+}$stress conditions. Deletion of Stb5 (4 potential binding sites in TRK1 and 10 in TRK2 promoter region) was, according to the distribution pattern expected to impact mainly on the low affinity TRK2 expression. The $\Delta$ stb5 mutant did grow slower than a $\Delta$ TRK2 disruption (entire $K^{+}$uptake via TRK1), indicating that even a reduced (up) regulation of TRK2 has, with a present TRK1, only a minor impact. The $\Delta$ mot3 strain exhibited a growth defect under $K^{+}$limiting conditions of approximately half the effect of disruption of the potassium transporter Trk1p. Mot3p is known as involved in repression of several 
DAN/TIR genes during aerobic growth, and repression of ergosterol biosynthetic genes. Under the assumption of a direct influence of Mot3p the lack of this TF could be responsible for a reduced expression of TRK1 (13 potential binding sites in TRK1 versus 7 in TRK2). However, alternatively, given the described role of Mot3p in ergosterol biosynthesis pathway, the deletion of this TF might pose an altered -for $\mathrm{K}^{+}$uptake transporters unfavourable- lipid composition onto the plasma membrane. In summary, the results from these initial phenotypic experiments are indeed interesting and hold the potential for extension towards construction and analysis of yeast strains bearing combinations of TF and potassium transporter mutations.

\section{Experiment 2}

No significant differences were found for $\mathrm{BMH} 1$ and $\mathrm{BMH} 2$ gene expression in the tested TFs mutants However, a regulation cannot be excluded. In addition, other TFs may take over regulatory role of the ones tested in this study. Another possibility is that the tested TFs are not active during exponential growth in a rich medium like YPD. In conclusion, we were not able to show an effect on regulation of BMH1 and BMH2 in the TF mutants tested in this study.

\section{Acknowledgments}

This work was supported by the German Ministry for Eduction and Research (BMBF grant for the SysMO Eranet project Translucent) and by the German Research Foundation (DFG grant for the International Research Training Group "Genomics and Systems Biology of Molecular Networks").

\section{References}

[1] Bertl, A., et al., Characterization of potassium transport in wild-type and isogenic yeast strains carrying all combinations of TRK1, TRK2 and TOK1 null mutations, Mol Microbiol, 47(3):767-780, 2003.

[2] Brachmann, C.B., Davies, A., Cost, G.J., Caputo, E., Li, J., Hieter, P., Boeke, J.D., Designer deletion strains derived from Saccharomyces cerevisiae S288C: a useful set of strains and plasmids for PCR-gene disruption and other applications, Yeast, 14:115-132, 1998.

[3] Gaber, R.F., et al. TRK1 encodes a plasma membrane protein required for high-affinity potassium transport in Saccharomyces cerevisiae, Mol Cell Biol, 8(7):2848-2859, 1988.

[4] Giaever, G., et al., Functional profiling of the Saccharomyces cerevisiae genome., Nature, 25(418)(6896):387-391,2002

[5] Hasenbrink, G., Schwarzer, S., Kolacna, L., Ludwig, J., Sychrova, H., Lichtenberg-Fraté, H., Analysis of the mKir2.1 channel activity in potassium influx defective strains determined as changes in growth characteristics, FEBS Letters, 579(7):1723-1731, 2005.

[6] Hertz, G.Z., Stormo, G,D., Identifying DNA and protein patterns with statistically significant alignments of multiple sequences, Bioinformatics, 15(7-8):563-577, 1999. 
[7] Hobby, J.D., A User's Manual for MetaPost, Tech. Rep. 162, AT\&T Bell Laboratories, Murray Hill, New Jersey, 1992.

[8] Hollenhorst, P.C., Bose, M.E., Mielke, M.R., Mller, U., Fox, C.A., Forkhead genes in transcriptional silencing, cell morphology and the cell cycle. Overlapping and distinct functions for FKH1 and FKH2 in Saccharomyces cerevisiae, Genetics, 154(4):1533-1548, 2000.

[9] Hong, E.L., Balakrishnan, R., Christie, K.R., Costanzo, M.C., Dwight, S.S., Engel, S.R., Fisk, D.G., Hirschman, J.E., Livstone, M.S., et al., Saccharomyces Genome Database, http://www. yeastgenome.org/, 2007.

[10] Hughes, J.D., Estep, P.W., Tavazoie, S., Church, G.M., Computational identification of cis-regulatory elements associated with groups of functionally related genes in Saccharomyces cerevisiae, J Mol Biol., 296(5):1205-1214, 2000.

[11] Ko, C.H., et al., TRK2 is required for low affinity $\mathrm{K}^{+}$transport in Saccharomyces cerevisiae, Genetics, 125(2):305-312, 1990.

[12] Ko, C.H., Gaber, R.F., TRK1 and TRK2 encode structurally related $K^{+}$transporters in Saccharomyces cerevisiae, Mol Cell Biol, 11(8):4266-4273, 1991.

[13] Livak, K.J., Schmittgen, T.D., Analysis of relative gene expression data using real-time quantitative PCR and the 2(-Delta Delta $\mathrm{C}(\mathrm{T})$ ) Method, Methods, 2(4):402-408, 2001.

[14] Martinez-Pastor, M.T., et al., The Saccharomyces cerevisiae zinc finger proteins Msn2p and Msn4p are required for transcriptional induction through the stress response element (STRE), EMBO J 15(9):2227-2235, 1996.

[15] Pevzner, P.A., Sze, S.H., Combinatorial approaches to finding subtle signals in DNA sequences, Proc Int Conf Intell Syst Mol Biol., 8:269-278, 2000.

[16] Schug, J., Overton, G.C., TESS: Transcription Element Search Software on the $W W W$, Tech. Rep. CBIL-TR-1997-1001-v0.0, Computational Biology and Informatics Laboratory, School of Medicine, University of Pennsylvania, 1997.

[17] Teixeira, M.C., Monteiro, P., Jain, P., Tenreiro, S., Fernandes, A.R., Mira, N.P., Alenquer, M., Freitas, A.T., Oliveira, A.L., et al., The YEASTRACT database: a tool for the analysis of transcriptional relatory associations in Saccharomyces cerevisiae, Nucleic Acids Res., 34 (Database Issue), D446-D451, 2006.

[18] Tompa, M. et al, Assessing computational tools for the discovery of transcription factor binding sites, Nature Biotechnology, 23: 137-144, 2005.

[19] The Gene Ontology Consortium, Gene Ontology: tool for the unification of biology, Nat. Genet., 25(1): 25-29, 2000.

[20] van Helden, J., Regulatory sequence analysis tools, Nucleic Acids Res., 31:3593-3596, 2003.

[21] van Heusden, G.P., Steensma, H.Y., Yeast 14-3-3 proteins, Yeast, 23(3):159-171, 2006.

[22] van Heusden, G.P., 14-3-3 proteins: Insights from genome-wide studies in yeast, Genomics, [Epub ahead of print], 2009.

[23] Wingender, E., Chen, X., Fricke, E., Geffers, R., Hehl, R., Liebich, I., Krull, M., Matys, V., Michael, H., et al., The TRANSFAC system on gene expression regulation, Nucleic Acids Res., 29:281-283, 2001.

[24] Yenush, L., et al., pH-Responsive, posttranslational regulation of the Trk1 potassium transporter by the type 1-related Ppz1 phosphatase, Mol Cell Biol, 25(19):8683-8692, 2005.

[25] http://www.genomatix.de/

[26] http://www. mathworks.com/

[27] http://www.cbil. upenn.edu/tess

[28] http://www.yeastract.com/ 